\title{
ECOSYSTEM ADAPTATION: DO ECOSYSTEMS MAXIMIZE RESILIENCE?
}

\author{
Roger Cropp ${ }^{1}$ AND Albert Gabric \\ School of Australian Environmental Studies, Griffith University, Nathan, Queensland 4111, Australia
}

\begin{abstract}
The response of an ecological system to perturbation can be described in terms of its resilience, essentially a measure of the time the system takes to return to its prior state. The resilience of an ecosystem is the result of interactions of the biota and their environment and will therefore change as the biota evolve and environmental conditions change. Ecological systems exist within the constraints of thermodynamic laws that prescribe the transfer of energy. Ecologically defined "thermodynamic imperatives," such as entropy, exergy, and ascendency, provide whole-ecosystem selection pressures that constrain the evolution of individuals within an ecosystem in addition to the selection pressures of individual evolution. The essence of these whole-ecosystem selection pressures may be captured by metrics. We have used a "genetic algorithm" to optimize these metrics, simulating the adaptation of a model ecosystem biota. Our simulations suggest the hypothesis that, within the constraints of the external environment and the genetic potential of their constituent biota, ecosystems will evolve to the state most resilient to perturbation.
\end{abstract}

Key words: ecosystem adaptation; nutrient enrichment; resilience; thermodynamic selection pressures.

\section{INTRODUCTION}

Aquatic ecosystems are being increasingly subjected to anthropogenic perturbation (Smith et al. 1999), with these perturbations often occurring in the form of increased nutrient loadings (Vitousek et al. 1997). The resilience of an ecosystem is a measure of how quickly the system returns to its prior state after a perturbation (Begon et al. 1996), and it is important that we understand this property of ecosystems. It is also critical that explanations we deduce for ecosystem-level properties are consistent with natural selection at the individual level (Lenton 1998).

Although difficult to measure in real ecosystems, resilience is an easily derived attribute of simple ecosystem models (DeAngelis 1992). Many aspects of ecosystems are simplified or idealized when we formulate models, and we cannot expect such simple models to simulate all the intricacies of real ecosystems. Simple models may, however, lead to a deeper understanding of the modeled phenomena (Bazykin 1998).

The overarching themes that shape ecosystem adaptation have been a topic of ecological research since Lotka (1922) suggested that natural selection would operate to preserve organisms that increased the total energy flux through a system. Common to many hypothesized ecosystem goal functions are underlying thermodynamic considerations about how ecosystems utilize the energy that flows through them. Ecosystems exist within the constraints of thermodynamic laws that prescribe the transfer of energy and may be considered as thermodynamic nonequilibrium dissipative structures

Manuscript received 8 December 2000; revised 5 April 2001; accepted 26 April 2001; final version received 17 October 2001.

${ }^{1}$ E-mail: R.Cropp@mailbox.gu.edu.au that, in common with their physical counterparts such as hurricanes and Bénard cells, utilize energy fluxes from external sources to maintain organization (Prigogine and Stengers 1984, Toussaint and Schneider 1998). Reviews of the current state of thermodynamics relating to ecology can be found in Jorgensen $(2000 a, b)$.

We have formulated simple metrics that capture the influence of these thermodynamic and ecological measures on a model aquatic ecosystem. These metrics are used as selection pressures to constrain the evolution of the individuals within the ecosystem. We have simulated the adaptation of the model ecosystem's biota to these selection pressures by coupling the ecosystem model to a genetic algorithm (GA), an efficient, nonlinear optimization technique based on Darwinian evolution (Holland 1975). This approach has the advantage of determining the biotic attributes of an ecosystem from fundamental properties, an alternative to the usual method of matching the model with empirical data (Jorgensen 1999).

Our thermodynamically constrained adaptation simulations generate an hypothesis of ecosystem evolution. This hypothesis leads to some counterintuitive implications for attributes that we might find in real ecosystems. Although based on a simple model and somewhat fuzzy thermodynamic concepts, there appears to be considerable empirical support for the hypothesis.

\section{Methods}

The essence of our approach lies in the coupling of a deterministic differential equation ecosystem model of population dynamics over non-evolutionary time scales with a stochastic GA that simulates individual adaptation in response to selection pressures over evolutionary time scales. The coupling between the models 
TABLE 1. Model equations, steady state, and ranges of parameter values.

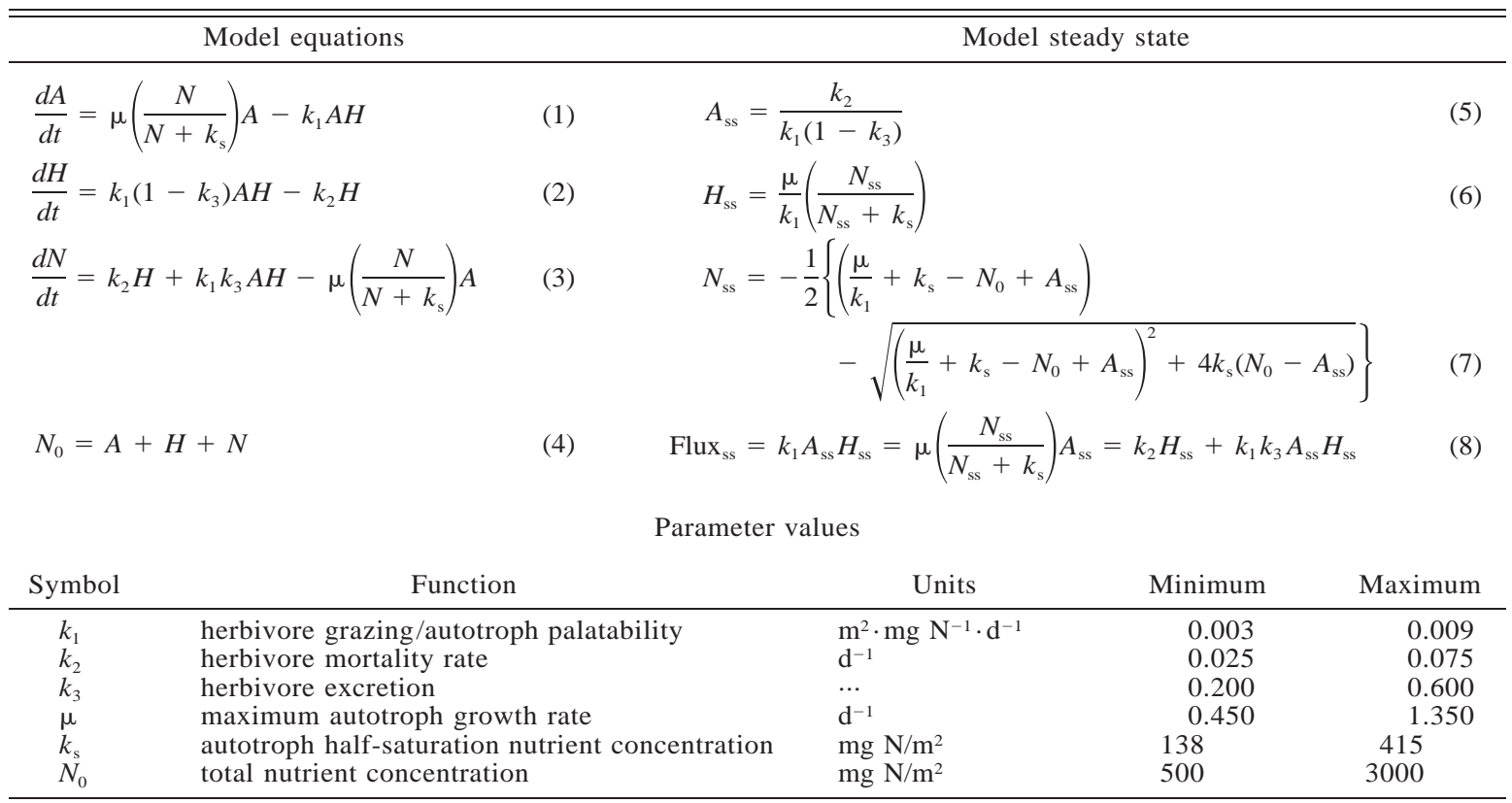

Note: Values are averages for a $20 \mathrm{~m}$ deep, mixed-layer oceanic environment.

was achieved by using selection pressures formulated from the ecosystem model as goal functions for the genetic algorithm.

A simple ecosystem model was used in this initial work, as we required a model that had a single, stable equilibrium point so that analytical expressions could be derived for the steady state selection pressures. The use of a simple model, while ignoring the "fine detail" of ecosystem function, does not preclude the model from simulating fundamental properties of ecosystems. We find merit in the arguments of Closs et al. (1999) that simple models that make clear predictions are more likely to advance ecological theory than highly complex models.

We used a simple autotroph-herbivore-nutrient (AHN) mixed-layer ecosystem model, based on standard formulations that are widely used in plankton modeling (McCauley and Murdoch 1990, DeAngelis 1992, Edwards and Brindley 1999, Huisman and Weissing 1999). The biomasses ( $A$ and $H$ ) and the mass of nutrient $(N)$ in the model are expressed as concentrations of nutrient, rather than numbers of individuals. The model differential equations (Table 1) conserve the total mass of nutrient $\left(N_{0}\right)$ of the ecosystem.

The model has five physiological parameters $\left(k_{1}\right.$, $\ldots, k_{\mathrm{s}}$ ) that define a five-dimensional parameter space (Table 1). We used empirical values reported for marine phytoplankton, zooplankton, and nitrogen (Moloney et al. 1986) for the centers of the parameter ranges and defined the extremes of the ranges as $\pm 50 \%$ of each of the reported values. Simulations were undertaken for two scenarios, a low-nutrient state $\left(N_{0}=500 \mathrm{mg}\right.$ $\left.\mathrm{N} / \mathrm{m}^{2}\right)$ and a high-nutrient state $\left(N_{0}=3000 \mathrm{mg} \mathrm{N} / \mathrm{m}^{2}\right)$.

The model has three finite steady states, although only one finite steady state is physically feasible (Table 1). The unstable steady state at $(A, H, N)=(0,0,0)$ is ignored as a trivial solution, and the steady state specified by the other root of the quadratic for $N_{\mathrm{ss}}$ is ignored as unrealistic as it specifies negative concentrations. Although the physically realistic steady state has several possible behaviors, within the parameter ranges defined for this study the steady state is always an asymptotically stable node. It is reactive in the sense of Neubert and Caswell (1997), and the model therefore responds to almost all perturbations by initially amplifying the magnitude of the perturbation, after which the perturbation envelope exponentially decays as the model returns to its steady state.

The resilience of an ecosystem model is defined mathematically as the negative of the real part of the dominant eigenvalue of the linearized system (DeAngelis 1980). This definition of resilience is strictly correct only for small perturbations from the steady state, but has proved to be a useful indicator of system responses to large perturbations (DeAngelis 1992). The expression for the resilience of the AHN model is given in Table 2.

The parameter space permits a range of ecosystem behaviors, from systems that eliminate the effects of very large perturbations almost immediately to ones that are extremely sensitive to perturbations. These latter systems do not recover from even small perturba- 
TABLE 2. Selection pressures used in the adaptation simulations.

\begin{tabular}{|c|c|c|}
\hline Selection pressure & Metric & Significance \\
\hline $\max A_{\mathrm{ss}}$ & $\frac{k_{2}}{k_{1}\left(1-k_{3}\right)}$ & $\begin{array}{l}\text { Maximum sustainable autotroph biomass. Maximum autotroph exergy } \\
\text { (Jorgensen 1992). }\end{array}$ \\
\hline $\max A_{\mathrm{tr}}$ & $\frac{1}{T} \int_{0}^{T} A \mathrm{dt}$ & $\begin{array}{l}\text { Maximum average autotroph biomass/exergy over the first transient after } \\
\text { a perturbation. } \dagger\end{array}$ \\
\hline $\max H_{\mathrm{ss}}$ & $\frac{\mu}{k_{1}}\left(\frac{N_{\mathrm{ss}}}{N_{\mathrm{ss}}+k_{\mathrm{s}}}\right)$ & $\begin{array}{l}\text { Maximum sustainable herbivore biomass. Also maximum herbivore ex- } \\
\text { ergy. }\end{array}$ \\
\hline $\max H_{\mathrm{tr}}$ & $\frac{1}{T} \int_{0}^{T} H \mathrm{dt}$ & $\begin{array}{l}\text { Maximum average herbivore biomass/exergy over the first transient after } \\
\text { a perturbation. } \dagger\end{array}$ \\
\hline $\max$ Flux $_{\mathrm{ss}}$ & $\mu\left(\frac{N_{\mathrm{ss}}}{N_{\mathrm{ss}}+k_{\mathrm{s}}}\right) A_{\mathrm{ss}}$ & $\begin{array}{l}\text { Maximum gross primary production. Maximum ascendency (Ulanowicz } \\
\text { 1980). }\end{array}$ \\
\hline $\max _{\text {Flux }}$ & $\frac{1}{T} \int_{0}^{T} \mu\left(\frac{N}{N+k_{\mathrm{s}}}\right) A \mathrm{dt}$ & Maximum average Flux over the first transient after a perturbation. $\dagger$ \\
\hline $\max P / B_{\mathrm{ss}}$ & $\frac{\text { Flux }_{\mathrm{ss}}}{\left(A_{\mathrm{ss}}+H_{\mathrm{ss}}\right)}$ & $\begin{array}{l}\text { Maximum gross primary production per unit biomass, power capacity } \\
\text { (Odum and Pinkerton 1955), entropy production (Schneider 1988), } \\
\text { or dissipation (Johnson 1988) at steady state. }\end{array}$ \\
\hline $\max P / B_{\mathrm{tr}}$ & $\frac{1}{T} \int_{0}^{T} \mu\left(\frac{N}{N+k_{\mathrm{s}}}\right)\left(\frac{A}{A+H}\right) \mathrm{dt}$ & Maximum average $P / B$ over first transient after a perturbation. $\dagger$ \\
\hline max resilience & $\frac{\mu}{2}\left(\frac{k_{\mathrm{s}}}{\left(N_{\mathrm{ss}}+k_{\mathrm{s}}\right)^{2}}\right) A_{\mathrm{ss}}$ & Maximum resilience (DeAngelis 1992). \\
\hline
\end{tabular}

Notes: Abbreviations are: ss, the steady state of the system; tr, transient state of the system. $A, H$, and $N$ are instantaneous values of the state variables; $B(=A+H)$ is total biomass; $T$ is the period of oscillation of the system; $t$ is time.

$\dagger$ All perturbations removed half of each of the autotroph and herbivore steady-state biomasses.

tions within an ecologically realistic time frame (i.e., several years). The reactivity inherent in the model indicates that such sensitive systems will often exhibit large amplitude, persistent oscillations (population booms and busts) in response to very small perturbations.

We simulated the evolutionary adaptation of the ecosystem model by coupling it to a GA. The GA utilizes the rules of evolution with natural selection, including random mutation, random crossing-over of genetic material during reproduction, and reproductive success proportional to fitness, to maximize the fitness of virtual chromosomes in a population with respect to a specified goal function (see Appendix for details). In our case, each GA chromosome represents one set of the five parameter values. The coupling was achieved by using selection pressures defined for the AHN ecosystem model as the goal functions of the GA.

Four basic selection pressures were formulated by considering thermodynamic (entropy, exergy, and ascendency) and ecological (sustainable biomass, primary productivity, and productivity per unit biomass) imperatives that influence ecosystems (Table 2). Although the thermodynamic aspects of ecosystems are not yet well understood, the selection pressures they imply are consistent with ecological theory. Each selection pressure was evaluated both at steady state and as an average over the first transient of the system, resulting in a set of eight ecosystem goal functions to be optimized by the GA.

Some authors (e.g., Margalef 1968, Odum 1969) have suggested that ecosystems minimize the primary production per unit biomass $(P / B)$. We elected to maximize $P / B$ in our selection pressures according with hypotheses of maximum power capacity (Lotka 1922, Odum and Pinkerton 1955) and entropy production (Johnson 1990). We will demonstrate that the two views are not incompatible, as our model ecosystem maximizes $P / B$ over evolutionary time, but minimizes $P / B$ over shorter time scales, such as when responding to a perturbation.

The resilience of the system was optimized similarly to the other goal functions (Table 2). Although there is little thermodynamic or ecological evidence to suggest that this is a legitimate selection pressure, ecological networks that develop stabilizing feedbacks are considered to be more likely to remain extant than those that do not (Lenton 1998).

Table 2 clearly shows that, as might be expected in a simple model, the expressions for the steady state goal functions are closely related, with quantities such as the steady state autotroph biomass $\left(A_{\mathrm{ss}}\right)$ appearing in the flux, $P / B_{\mathrm{ss}}$, and resilience goal functions, as well as being a goal function in its own right. This should not however be taken as an indication that the goal functions are monotonically related to $A_{\mathrm{ss}}$. The steady 
state nutrient concentration $\left(N_{\mathrm{ss}}\right)$ appears in all the steady state goal functions apart from $A_{\mathrm{ss}}$, and as can be seen from Eq. 6 (Table 1), contains $A_{\mathrm{ss}}$. If the righthand side of Eq. 7 is substituted in the steady state goal functions in place of $N_{\mathrm{ss}}$, we can readily see that the goal functions are highly nonlinear functions of $A_{\mathrm{ss}}$ and in fact have multiple critical points. The goal functions are thus not necessarily convex hypersurfaces in the parameter space, and the parameter sets that optimize each of these functions are not necessarily vertices of the space.

The parameter space represents the genetic potential of the AHN system's biota to adapt in response to selection pressures. As the GA simulates the adaptation of the ecosystem, changes in the values of the five parameters can reflect either species shifts within a community or physiological adaptations by individual species. Ten simulations of the ecosystem's adaptation to each selection pressure were performed to investigate the sensitivity of the goal functions to each parameter. The means of the 10 simulations were used to indicate the "optimal" parameter values, and the coefficients of variation (CV) of the means were used as estimates of the sensitivity of the goal functions to each of the parameters. Parameters with CV's similar to that of the selection pressure were considered influential.

\section{RESULTS}

The adaptation simulations in a low-nutrient environment (Table 1, Appendix B) indicate that all the selection pressures are optimized at the vertex of the parameter space defined by

$$
\begin{aligned}
& k_{1}=0.003 \\
& k_{2}=0.075 \\
& k_{3}=0.600 \\
& \mu=1.350 \\
& k_{\mathrm{s}}=138 .
\end{aligned}
$$

Most of the selection pressures are unequivocally maximized at this vertex, although three $\left(H_{\mathrm{ss}}, H_{\mathrm{tr}}\right.$, and $P$ / $B_{\text {ss }}$ ) can achieve slightly higher maxima with the values of $k_{1}, k_{2}$, or $k_{3}$ indicated in Table 1 . These selection pressures are, however, only slightly sensitive to variations in these parameters, and the increases are of the order of only a few percent.

The optimizing parameter set (Eqs. 9a-e) describes a system with a rapidly growing, unpalatable autotroph subject to low grazing pressure of a herbivore that inefficiently converts ingested nutrient to biomass and experiences high mortality. The predicted autotroph attributes, of rapid growth and optimal nutrient utilization, accord with attributes expected from Darwinian evolution with individual selection pressures. The predicted herbivore attributes, however, are both counterintuitive and contrary to attributes that might be ex- pected from individual-based evolution. The herbivore attributes result in organisms that are less fit to compete for limiting resources at the individual level. It is also counterintuitive that the autotroph and herbivore biomasses can be maximized by noncontradictory parameter sets. Further, it is also unexpected, considering their substantially different mathematical formulations, that steady state and transient selection pressures should be sensitive to the same parameters and indeed are maximized by the same values.

The most interesting aspect of the simulation is that the biotic attributes that optimize the thermodynamic goal functions also maximize the system's resilience. The probability of nine independent functions being optimized at the same vertex of a five-dimensional parameter space is $2.8 \times 10^{-14}$ and substantially less if optima at interior points are considered. Steady-state relationships between $P / B$ and resilience have been demonstrated previously for Lotka-Volterra and Michaelis-Menten models (DeAngelis 1980, Moore et al. 1993), although Stone et al. (1996) showed that such relationships are not necessarily monotonic, even for simple Lotka-Volterra models. Our simulations imply these relationships might also apply to transient dynamics and also suggest additional relationships between biota biomasses and resilience.

There is also no a priori indication that the optimization of an ecosystem's response to thermodynamic or ecological imperatives should result in a maximally resilient ecosystem. This outcome, however, is reasonable, given that all ecosystems exist within the constraints of thermodynamic laws and that highly resilient ecosystems are more likely to remain extant than ecosystems with low resilience.

The adaptation simulations for a high-nutrient environment (Table 2, Appendix B) also reveal that a maximum resilience parameter set optimizes the set of thermodynamic goal functions:

$$
\begin{aligned}
& k_{1}=0.003 \\
& k_{2}=0.075 \\
& k_{3}=0.600 \\
& \mu=1.350 \\
& k_{\mathrm{s}}=415 .
\end{aligned}
$$

This parameter set is similar to the low-nutrient optimum parameter set, differing only in the value of $k_{\mathrm{s}}$. The resilience is highly sensitive to $k_{\mathrm{s}}$ (indeed to all parameters) in both simulations, with resilience being maximized by low values of $k_{\mathrm{s}}$ in low-nutrient systems and high values of $k_{\mathrm{s}}$ in high-nutrient systems. While four selection pressures in addition to resilience were sensitive to $k_{\mathrm{s}}$ in the low-nutrient simulations, only resilience was sensitive to $k_{\mathrm{s}}$ in the high-nutrient simulations.

Simulations maximizing resilience for nutrient load- 
ings between the low-nutrient and high-nutrient extremes, with $k_{\mathrm{s}}$ allowed to vary over the entire nutrient range, were also undertaken. Optimum points were then found at interior (i.e., non-vertex) points of the parameter space. Regression analysis revealed a strong linear relationship between the total nutrient $\left(N_{0}\right)$ and the value of $k_{\mathrm{s}}$ that maximized the resilience of the system

$$
\begin{aligned}
& k_{\mathrm{s}}=0.99 \times N_{0}-497 \quad R^{2}=0.9997 \\
& p \ll 0.001
\end{aligned}
$$

The trend to increased phytoplankton $k_{\mathrm{s}}$ values in response to increased ambient nutrient concentrations may be considered an "emergent property" of the maximally resilient system.

\section{DISCUSSION}

Our results are based on the evolution of a simple ecosystem model under the constraint of thermodynamic and ecological goal functions that as yet have little rigorous basis. A measure of the validity of our results may be obtained by considering how the results of our simulations compare with properties of real ecosystems.

A significant result of our simulations is the emergence of the relationship between the autotroph halfsaturation constant $k_{\mathrm{s}}$ and the resilience of the ecosystem. Empirical investigations have documented that phytoplankton $k_{\mathrm{s}}$ values do vary with ambient nutrient concentration (Eppley et al. 1969, MacIsaac and Dugdale 1969, Carpenter and Guillard 1971). The values of $k_{\mathrm{s}}$ predicted by our regression agree with measurements of marine phytoplankton $k_{\mathrm{s}}$ values for nitrate and ammonium of $0-28 \mathrm{mg} \mathrm{N} / \mathrm{m}^{2}$ for low-nutrient conditions in the Sargasso Sea to $2213-3557 \mathrm{mg} \mathrm{N} / \mathrm{m}^{2}$ for high-nutrient conditions at La Jolla (Eppley et al. 1969).

Further, our predictions that highly resilient ecosystems will have low values of $k_{1}$ and high values of $k_{3}$ agree with two ecosystem-stabilizing effects that have recently been hypothesized from studies of real plankton ecosystems (McCauley et al. 1999). From studies of algae-Daphnia-nutrient ecosystems in lakes and microcosms, McCauley et al. concluded that the addition of inedible algae could stabilize the system, even in high-nutrient environments. The addition of inedible algae is analogous to a reduction of $k_{1}$ in our model, as this parameter reflects the "average palatability" of the algal assemblage. They further noted that the systems could also be stabilized if Daphnia's rate of increase was reduced by the production of ephippia, resting eggs that require substantial energy input but do not contribute immediately to population growth. In our model, such a physiological response is analogous to an increase in $k_{3}$, the proportion of nutrient consumed by the herbivores that does not contribute to population growth.

The maximally resilient system predicted by our sim- ulations also reflects the production per unit biomass $(P / B)$ characteristics of real ecosystems. When the maximum resilience system is perturbed from its steady state, for example by a reduction of its biomass, the system responds by very rapidly increasing $P / B$ to a level well above its steady-state value, after which $P / B$ reduces until it returns to its steady-state level. This behavior has been observed in mesocosm experiments in which ecosystems were subject to perturbations (pollution) that moved them away from their steady state by reducing the biomass of the system (Schneider 1988).

The concordance of ecosystem attributes predicted by our simulations with attributes observed in real ecosystems suggests that our model may be reflecting the mechanisms by which at least some ecosystems change. We consider that these results support our contention that our simple ecosystem adaptation model captures the fundamental properties of ecosystems and that our thermodynamic/ecological goal functions are feasible representations of the factors influencing ecosystem evolution.

The principal implication of our simulations is the hypothesis that ecosystems evolve to maximize resilience. This hypothesis concurs with the conclusions of Johnson's (1990) extensive study of isolated Arctic lake ecosystems. On the basis of his empirical work, Johnson proposed the hypothesis that "all autonomous ecosystems, irrespective of their diversity, tend to assume a stable state, within the boundary constraints of the system, the regularity of the energy input and the genetic make-up of their species populations" (Johnson 1990:9). Our results suggest that Johnson's hypothesis may be refined to contend that ecosystems will evolve to the most stable state available.

Testing ecosystem hypotheses is not a trivial task, and in this case the resilience of a real ecosystem is not readily measurable. The best approach to testing our hypothesis may lie in defining verifiable attributes we might expect to find in real ecosystems.

Our results suggest that the ratio $\mu / k_{1}$, which can be relatively easily obtained for real ecosystems, may be a useful surrogate measure of ecosystem resilience. This ratio reflects the coupling between the autotrophs and herbivores in an ecosystem, being a ratio of the growth attributes of the autotroph $(\mu)$ and the interaction of the autotroph palatability and herbivore grazing characteristics $\left(k_{1}\right)$. Our simulations imply that ecosystems with high values of $\mu / k_{1}$ will have high resilience to perturbation (Fig. 1). The nature of the relationship between $\mu / k_{1}$ and resilience is not clear, as linear, quadratic, power, and exponential functions all explain $>95 \%$ of the variation in the data in Fig. 1 . Paradoxically, high values of $\mu / k_{1}$ do not necessarily imply high steady-state autotroph biomass; in this model they result instead in high steady-state herbivore biomass. Inversions of the classic trophic biomass pyramids such as this have been well documented in ponds 


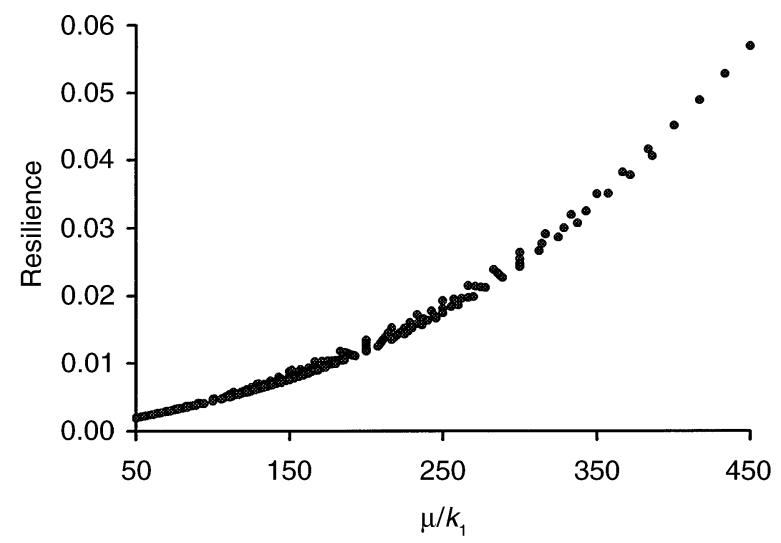

FIG. 1. Resilience of systems vs. $\mu / k_{1}$. Values of $\mu / k_{1}$ are obtained from various ratios of $0.45<\mu<1.35$ and 0.003 $<k_{1}<0.009(n=147)$. Other parameter values are for the maximum resilience system.

and are defined as the annual clear-water stage in many lacustrine environments (Straskraba et al. 1999).

Simulations with the maximum resilience ecosystem indicate that the sensitivity of the resilience to $k_{\mathrm{s}}$ varies according to the ambient nutrient level of the system. Comparison of the normalized resilience of an ecosystem adapted to a low-nutrient environment with the normalized resilience of the same (in the other four parameters) ecosystem adapted to intermediate and high-nutrient environments (Fig. 2) reveals that increasing the total nutrient of an ecosystem broadens the range of $k_{\mathrm{s}}$ values for which the resilience is close to its maximum. This suggests that we might expect to find a greater range of $k_{\mathrm{s}}$ values in a high-nutrient ecosystem and that the rate at which autotrophs will increase $k_{\mathrm{s}}$ in response to nutrient enrichments will slow as nutrient loadings increase.

Our hypothesis that ecosystems will adapt to maximize resilience does not imply that ecosystems will be impervious to perturbation. Simulations predict that model ecosystems that have adapted $k_{\mathrm{s}}$ to low-nutrient regimes can be severely destabilized (i.e., will have longer return times, the inverse of resilience; DeAngelis 1980) by the addition of nutrient (Fig. 3). The severity of the destabilization is therefore more dependent on the history of the nutrient regime of the ecosystem prior to perturbation than on the contemporary nutrient loading. This is of fundamental importance for managing the impact of nutrient enrichment, as it suggests we can expect the effects of anthropogenic nutrient addition on oligotrophic ecosystems, such as coral reefs, to be more severe than the equivalent nutrient addition to systems adapted to high nutrient. Similar hypotheses have been proposed from empirical studies of coral reef ecosystems (i.e., Bell and Elmetri 1995).

The short return times predicted for adapted systems at high-nutrient loadings in Fig. 3 also correspond with empirical observations that stable plankton populations, in the field and in mesocosms, are not restricted

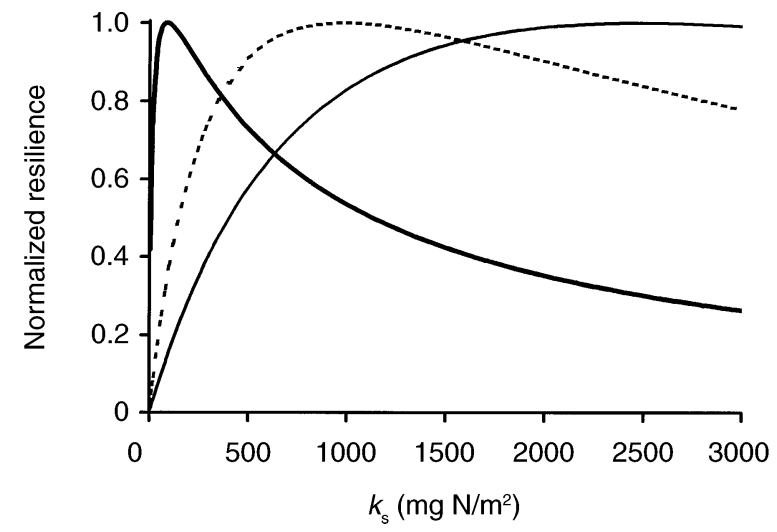

FIG. 2. The resilience, normalized to the maximum resilience for each nutrient level, of maximally resilient systems with total nutrient $\left(N_{0}\right)$ of $600 \mathrm{mg} \mathrm{N} / \mathrm{m}^{2}$ (thick line), $1500 \mathrm{mg}$ $\mathrm{N} / \mathrm{m}^{2}$ (dotted line), and $3000 \mathrm{mg} \mathrm{N} / \mathrm{m}^{2}$ (thin line) for different values of $k_{\mathrm{s}}$.

to low-nutrient systems (McCauley and Murdoch 1990). These authors also note that the destabilizing effect of nutrient enrichment is a fundamental property of a large range of predator-prey models, including the one we have used. It is particularly interesting, therefore, that the evolutionary pressures we postulate lead to the prediction of relatively stable states for the ecosystem model, even when subject to nutrient enrichment. Our hypothesis also supports contentions (i.e., Jorgensen 1999) that unstable or chaotic dynamics will be uncommon in unperturbed ecosystems. High resilience is predicted for adapted ecosystems, although our simulations suggest that unstable or chaotic dynamics may be introduced into previously stable ecosystems by perturbations such as species invasions or extinctions, or nutrient enrichment.

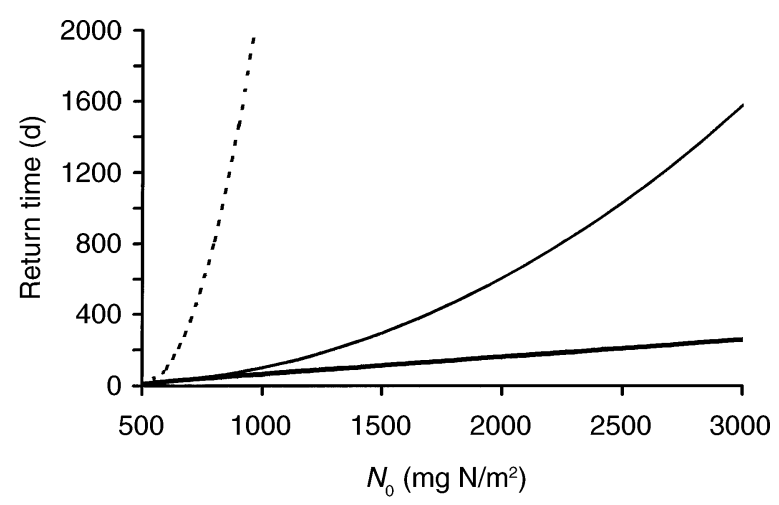

FIG. 3. A comparison of the predicted return times of maximally resilient systems for increasing nutrient loads. The thick line represents the resiliences of ecosystems that have $k_{\mathrm{s}}$ adapted to each nutrient load but have the same values for all other parameters. The dotted line represents the resilience of an ecosystem adapted to low nutrient $\left(N_{0}=500 \mathrm{mg} \mathrm{N} /\right.$ $\mathrm{m}^{2}$ ), and the thin line represents an ecosystem adapted to $N_{0}$ $=600 \mathrm{mg} \mathrm{N} / \mathrm{m}^{2}$. 


\section{Concluding remarks}

The principal outcome of our ecosystem adaptation simulations is the hypothesis that ecosystems evolve to the most stable state available within the constraints of their environments and the genetic potential of their constituent biota. We have documented empirical evidence that both supports the fundamental results of our simulations and suggests that the predictions we make on the basis of our hypothesis are reasonable.

The concurrence of our theoretical results and observed properties of real ecosystems further suggests that our thermodynamic imperatives, although yet to be rigorously defined in relation to living systems, may be reasonable representations of the constraints on ecosystem evolution. The analogous results of adaptation simulations of both steady state and transient selection pressures provide promise that applications of this approach may not be limited to models that have analytically tractable steady states. Future work to simulate thermodynamically constrained ecosystem evolution with more complex models will provide a more reliable indication of the generality of our results.

While our hypothesis relates to the evolution of ecosystems, our simulations do not have an intrinsic time scale. Changes in parameter values may also represent non-evolutionary ecosystem change such as shifts in species composition. The maximization of resilience may therefore be an emergent property of ecosystems apparent over a range of time scales.

\section{ACKNOWLEDGMENTS}

We wish to thank Roger Braddock, Rachel King, Roger Nisbet, Graham Sander, Lewi Stone, and an anonymous reviewer for their contributions to, and comments on, this work.

\section{Literature Cited}

Bazykin, A. D. 1998. Nonlinear dynamics of interacting populations. World Scientific, Singapore, Malaysia.

Begon, M., J. L. Harper, and C. R. Townsend. 1996. Ecology: individuals, populations and communities. Blackwell Science, Oxford, UK.

Bell, P. R. F., and I. Elmetri. 1995. Ecological indicators of large-scale nutrient enrichment in the Great Barrier Reef Lagoon. Ambio 24:208-215.

Carpenter, E. J., and R. R. L. Guillard. 1971. Intraspecific differences in nitrate half-saturation constants for three species of marine phytoplankton. Ecology 52:183-185.

Closs, G. P., S. R. Balcombe, and M. J. Shirley. 1999. Generalist predators, interaction strength and food web stability. Advances in Ecological Research 28:93-126.

DeAngelis, D. L. 1980. Energy flow, nutrient cycling and ecosystem resilience. Ecology 61:764-771.

DeAngelis, D. L. 1992. Dynamics of nutrient cycling and foodwebs. Chapman and Hall, London, UK.

Edwards, A. M., and J. Brindley. 1999. Zooplankton mortality and the dynamical behavior of plankton population models. Bulletin of Mathematical Biology 61:303-309.

Eppley, R. W., J. N. Rogers, and J. J. McCarthy. 1969. Halfsaturation constants for uptake of nitrate and ammonium by marine phytoplankton. Limnology and Oceanography 14:912-920.

Holland, J. H. 1975. Adaptation in natural and artificial sys- tems. University of Michigan Press, Ann Arbor, Michigan, USA.

Huisman, J., and F. J. Weissing. 1999. Biodiversity of plankton by species oscillations and chaos. Nature 402:407-410.

Johnson, L. 1988. The thermodynamic origin of ecosystems: a tale of broken symmetry. Pages 75-105 in B. H. Weber, D. J. Depew, and J. D. Smith, editors. Entropy, information and evolution. MIT Press, Cambridge, Massachusetts, USA.

Johnson, L. 1990. The thermodynamics of ecosystems. Pages 1-47 in O. Hutzinger, editor. The handbook of environmental chemistry: the natural environment and the biogeochemical cycles. Springer-Verlag, Berlin, Germany.

Jorgensen, S. E. 1992. Development of models able to account for changes in species composition. Ecological Modelling 62:195-208.

Jorgensen, S. E. 1999. State-of-the-art of ecological modelling with emphasis on the development of structural dynamic models. Ecological Modelling 120:75-96.

Jorgensen, S. E. 2000a. A general outline of thermodynamic approaches to ecosystem theory. Pages 113-133 in S. E. Jorgensen and F. Muller, editors. Handbook of ecosystem theories and management. CRC Press, Boca Raton, Florida, USA.

Jorgensen, S. E., editor. 2000b. Thermodynamics and ecological modelling. CRC Press, Boca Raton, Florida, USA.

Lenton, T. M. 1998. Gaia and natural selection. Nature 394: 439-447.

Lotka, A. J. 1922. Contribution to the energetics of evolution. Proceedings of the National Academy of Sciences (USA) 8:151-154.

MacIsaac, J. J., and R. C. Dugdale. 1969. The kinetics of nitrate and ammonia uptake by natural populations of marine phytoplankton. Deep Sea Research 19:45-57.

Margalef, R. 1968. Perspectives in ecological theory. University of Chicago Press, Chicago, Illinois, USA.

McCauley, E., and W. W. Murdoch. 1990. Predator-prey dynamics in environments rich and poor in nutrients. Nature 343:455-457.

McCauley, E., R. M. Nisbet, W. W. Murdoch, A. M. de Roos, and W. S. C. Gurney. 1999. Large-amplitude cycles of Daphnia and its algal prey in enriched environments. Nature 402:653-656.

Moloney, C. L., M. O. Bergh, J. G. Field, and R. C. Newell. 1986. The effect of sedimentation and microbial nitrogen regeneration in a plankton community: a simulation investigation. Journal of Plankton Research 8:426-445.

Moore, J. C., P. C. Ritter, and H. W. Hunt. 1993. Influence of productivity on the stability of real and model ecosystems. Science 261:906-908.

Neubert, M. G., and H. Caswell. 1997. Alternatives to resilience for measuring the responses of ecological systems to perturbations. Ecology 78:653-665.

Odum, E. P. 1969. The strategy of ecosystem development. Science 164:262-270.

Odum, H. T., and R. C. Pinkerton. 1955. Time's speed regulator: the optimum efficiency for maximum power output in physical and biological systems. American Scientist 43: 331-343.

Prigogine, I., and I. Stengers. 1984. Order out of chaos: man's new dialogue with nature. Bantam, New York, New York, USA.

Schneider, E. D. 1988. Thermodynamics, ecological succession and natural selection: a common thread. Pages $107-$ 138 in B. H. Weber, D. J. Depew, and J. D. Smith, editors. Entropy, information and evolution. MIT Press, Cambridge, Massachusetts, USA.

Smith, V. H., G. D. Tilman, and J. C. Nekola. 1999. Nutrient enrichment: impacts of excess nutrient inputs on fresh- 
water, marine and terrestrial ecosystems. Environmental Pollution 100:179-196.

Stone, L., A. Gabric, and T. Berman. 1996. Ecosystem resilience, stability and productivity: seeking a relationship. American Naturalist 148:892-903.

Straskraba, M., S. E. Jorgensen, and C. Patten. 1999. Ecosystems emerging: 2. Dissipation. Ecological Modelling 117:3-39.

Toussaint, O., and E. D. Schneider. 1998. The thermody- namics and evolution of complexity in biological systems. Comparative Biochemistry and Physiology Part A 120: 3-9.

Ulanowicz, R. E. 1980. An hypothesis on the development of natural communities. Journal of Theoretical Biology 85 : 223-245.

Vitousek, P. M., H. A. Mooney, J. Lubchenco, and J. M. Melillo. 1997. Human domination of Earth's ecosystems. Science 277:494-499.

\section{APPENDIX A}

A description of the genetic algorithm is available in ESA's Electronic Data Archive: Ecological Archives E083-036-A1.

\section{APPENDIX B}

Tables of parameter values that optimize selection pressures is available in ESA's Electronic Data Archive: Ecological Archives E083-036-A2. 